\title{
Diagnostic exome sequencing for patients with a family history of consanguinity: over $38 \%$ of positive results are not autosomal recessive pattern
}

\author{
Zöe Powis, Kelly D Farwell, Christina L Alamillo and Sha Tang
}

Diagnostic exome sequencing (DES) is an effective tool for diagnosis in intractable cases where the underlying cause is thought be genetic. It is commonly assumed that patients with a family history of consanguinity will have increased detection rates for rare autosomal recessive Mendelian disorders through DES. Herein, we analyzed the diagnostic yield and relevant inheritance patterns within the DES cases with a reported consanguineous family history. Of the first 500 unselected cases referred for DES, $40(8.0 \%)$ had a known consanguineous family history. Among the 40 cases, 13 (32.5\%) received a definitive molecular diagnosis through DES and such positive rate is similar to that of families with no reported consanguinity $(139 / 460,30.2 \%$, $P=0.63$ ). Although homozygous alterations likely related to consanguinity have been identified in eight positive cases, the other five $(38.4 \%)$ causative mutations were unrelated to autosomal recessive inheritance. Our retrospective analysis demonstrated that individuals with known consanguinity were not more likely to have a positive DES result and a significant portion of the positive findings were not within an autosomal recessive gene. These results highlight that all applicable inheritance patterns should be considered for patients with a known family history of consanguinity.

Journal of Human Genetics (2016) 61, 173-175; doi:10.1038/jhg.2015.125; published online 22 October 2015

\section{INTRODUCTION}

The practice of marriage and childbearing between closely related individuals is a widespread historical practice not limited to specific populations or religions. ${ }^{1}$ Although consanguinity may be practiced for cultural, religious or economic reasons, some studies report the risk of birth defects and genetic conditions higher in children of consanguineous parents due to increased risk for biallelic identity-bydescent disease alleles. ${ }^{2,3}$ For example, a report of an extra $1.7-2.8 \%$ risk for congenital anomalies in progeny of first cousin marriage. ${ }^{4}$

Since 2011, diagnostic exome sequencing (DES) has been shown to be an effective tool for diagnosis in intractable cases where the underlying cause is thought to have Mendelian inheritance. Recent journals have numerous case reports of individuals with consanguineous family histories in whom rare genetic conditions are identified by DES. ${ }^{5-8}$ It is commonly assumed that patients with a family history of consanguinity will have increased detection rates for rare autosomal recessive (AR) disorders through DES.

Small studies have focused upon DES as a tool for diagnosis for consanguineous families. Makrythansis et al. ${ }^{9}$ reported findings of array and exome studies of 50 consanguineous Arab families with two individuals with presumed genetic disorders. Seventeen families were found to have homozygous mutations. In a blinded study, Teeuw et al. $^{4}$ performed exome sequencing on four families with previously identified heterozygous alteration carrier parents and their homozygous affected children to determine if sequencing would pick up these alterations. Other studies have shown that genetic disorders within consanguineous families are not necessary due to AR conditions, demonstrating the need to look beyond basic assumptions. ${ }^{10}$

To date, studies have focused upon AR inheritance in the diagnosis of Mendelian genetic disorders with DES in individuals with consanguineous parents, recently using autozygosity mapping coupled with DES to identify potential alterations. Researchers using this method report that it is flawed and misses diagnoses. ${ }^{11,12}$ Herein, we report results from patients referred to Ambry Genetics (Aliso Viejo, CA, USA) for DES with a reported consanguineous family history without bias to inheritance pattern.

\section{MATERIALS AND METHODS}

Patients were ascertained sequentially using clinical samples sent to Ambry Genetics Laboratory for DES beginning September, 2011. Solutions Institutional Review Board determined the study to be exempt from the Office for Human Research Protections Regulations for the Protection of Human Subjects (45 Code of Federal Regulations 46) under category 4. Using retrospective data analysis of anonymized data exempted the study from the requirement to receive consent from patients.

Detailed methodology for exome sequencing, analysis and criteria for clinical interpretation has previously been described. ${ }^{13}$ Families with a reported consanguineous family history were retrospectively reviewed for ethnicity, degree of consanguinity, diagnostic yield and inheritance patterns for positive findings. A positive or causative finding was defined as a deleterious alteration (s) with significant gene overlap and consistent disease mechanism (gain of 


\section{Inheritance Patterns among Patients with Reported Consanguinity and Positive DES Result}

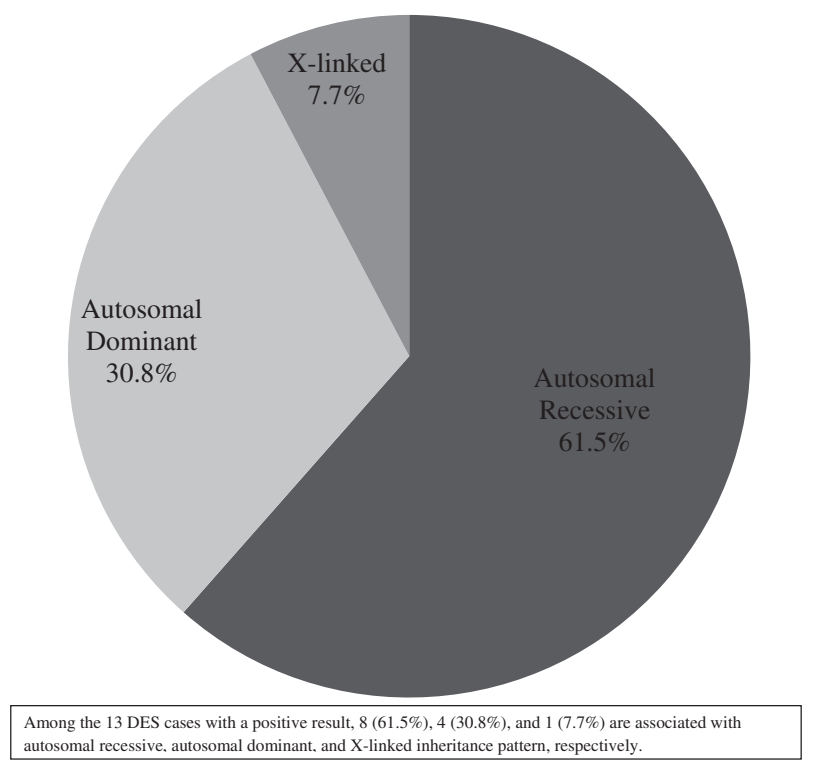

\section{Inheritance Patterns among Patients without Reported Consanguinity and Positive DES Result}

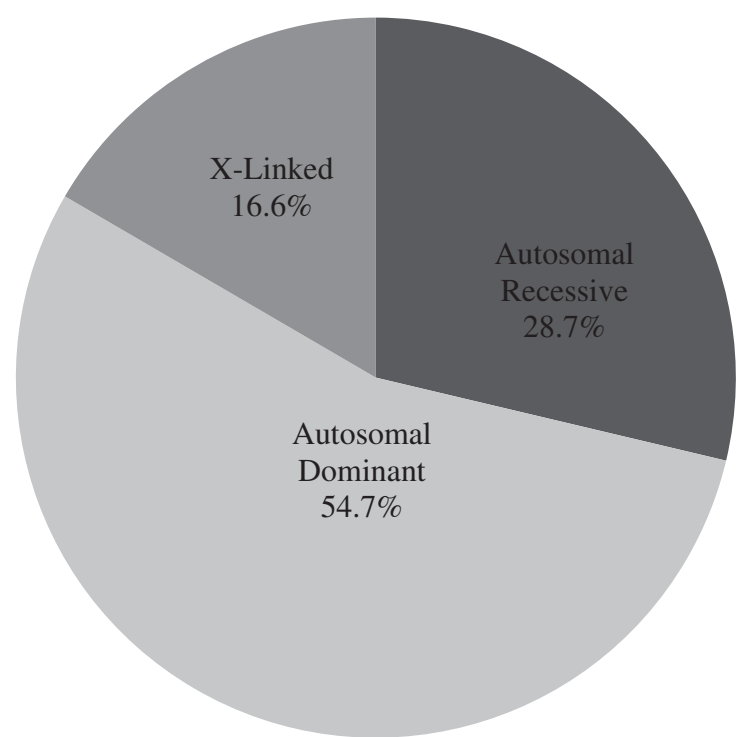

Among the 150 DES cases without consanguinity with a positive result, 43 (28.7\%), 82 $(54.7 \%)$, and $25(16.6 \%)$ are associated with autosomal recessive, autosomal dominant, and Xlinked inheritance pattern, respectively

Figure 1 Inheritance patterns among patients with reported consanguinity and positive DES result and patterns among patients without reported consanguinity and positive DES result. Among the 13 DES cases with a positive result, $8(61.5 \%), 4(30.8 \%)$ and $1(7.7 \%)$ are associated with autosomal recessive (AR), autosomal dominant and X-linked inheritance pattern, respectively. Among the $150 \mathrm{DES}$ cases without consanguinity with a positive result, $43(28.7 \%), 82(54.7 \%)$ and $25(16.6 \%)$ are associated with autosomal recessive, autosomal dominant and X-linked inheritance pattern, respectively.

function versus loss of function) as previously described ${ }^{13}$ (Supplementary Figure 1). Families with denied or unknown consanguinity were excluded. Statistical analysis was performed using Fisher's exact test.
Table 1 Positive gene finding, inheritance pattern, and degree of relationship among the families with reported consanguinity and a positive DES result

\begin{tabular}{llll}
$\begin{array}{l}\text { Family } \\
\#\end{array}$ & $\begin{array}{l}\text { Positive gene } \\
\text { finding }\end{array}$ & Inheritance & Degree of relationship \\
\hline 1 & ARHGEF9 & Heterozygous, de novo & Second cousins \\
2 & CHD7 & Heterozygous, de novo & First cousins \\
3 & MPZ & Heterozygous, de novo & Second cousins once removed \\
4 & TRPS1 & Heterozygous, de novo & Distant cousins \\
5 & VPS35 & Heterozygous, unknown & Unknown \\
6 & ACAT1 & Homozygous, inherited & First cousins \\
7 & ALS2 & Homozygous, inherited & First cousins once removed; \\
& & Homozygous, inherited & First cousins \\
8 & NEB & Homozygous, inherited & First cousins \\
9 & PDE6B & Homozygous, inherited & First cousins \\
10 & SPAST & Homozygous, inherited & At least double 1st cousins \\
11 & TCIRG1 & Homozygous, inherited & Second cousins \\
12 & TMEM231 & Homozygous, inherited & Second cousins once removed \\
13 & UBE3B & Hogree &
\end{tabular}

Degree of relationship is based on the pedigree and/or clinical notes provided.

\section{RESULTS}

Of the first 500 unselected cases referred for DES, 40 (8.0\%) had a reported consanguineous family history. The degree of consanguinity varied, including uncle and niece or double first cousin relationships $(F=1 / 8)$, first cousins $(F=1 / 16)$, multiple loops of consanguinity and 'distant consanguinity'. Clinicians reported 'Hispanic' ancestry in six cases $(17.5 \%$ of cases with a reported ancestry, race or country of origin. The additional most frequent countries of origin reported were Pakistan (five cases), India (four cases), Yemen (three cases) and reported 'Middle Eastern' ancestry represent $54.3 \%$ of the consanguinity families in this group (19/35 families with country of origin information provided).

The degree of consanguinity was not found to increase the likelihood of a positive result, with a mean of $F=1 / 16$ for both positive and negative result groups. Among the 40 families with a history of consanguinity, 13 had a positive result. These results were compared with the detection rates in the overall first 500 cases and was found to not be statistically significant ( $P=0.63$, Fisher's exact test) (Supplementary Table 1).

Of the positive results, $8(61.5 \%)$ had homozygous mutations in AR genes (ACAT1, ALS2, NEB, PDE6B, SPAST, TCIRG1, TMEM231 and $U B E 3 B), 4(30.7 \%)$ had alterations in autosomal dominant genes (VPS35, MPZ, CHD7 and TRPS1) and 1 alteration (7.7\%) was identified in an X-linked gene (ARHGEF9). Four of the five non-AR mutations (CHD7, MPZ, ARHGEF9 and TRPS1) arose de novo (Figure 1, Table 1).

\section{DISCUSSION}

Traditionally, it has been believed that individuals with known consanguinity would be more likely to have a positive result within an AR gene. Our retrospective analysis of the first 500 cases received for DES demonstrated that this was not the case. First of all, there were no statistical differences in detection rates between consanguineous versus non-consanguineous families, indicating that family history of consanguinity, even with high coefficient of inbreeding, is not a powerful determinant to predict the possibility of a diagnosis through DES. More interestingly, our data showed that almost 40\% (38.4\%, autosomal dominant and X-linked patterns combined) of the DES 
positive findings are actually unrelated to identity-by-descent and molecular diagnosis made through DES, especially those related to de novo mutations, dramatically changed the genetic counseling and family planning for related families. The recurrent risk dropped from a prior estimate of about $25-<1 \%$ (assuming no parental germline mosaicism).

The high ratio of positive findings unrelated to $A R$ inheritance in our study is most likely over-estimated. Some patients with consanguineous parents may have previous informative single gene or gene panel testing results guided by clinical evaluation and/or single nucleotide polymorphism (SNP) array analysis to narrow down the region of heterozygosity $(\mathrm{ROH})$ regions. Only patients with unrevealing previous molecular tests navigated the diagnostic odyssey through DES. At this time, the current state of DES focuses upon Mendelian diseases and not oligogenic disease or modifier mutations. A larger, more stratified study with more unbiased inclusion of patients will be valuable to better delineate the diagnostic yield and distribution of Mendelian inheritance patterns when DES is used as the first line of molecular testing.

Although counseling may have previously focused on AR inheritance patterns, all inheritance patterns should be discussed with consanguineous families. It is easy to become blinded by AR conditions when evaluating those with a reported family history of consanguinity; however, these results highlight that all applicable inheritance patterns should be considered when testing patients with a known family history of consanguinity.

\section{CONFLICT OF INTEREST}

All authors are employed in Ambry Genetics and received salary from Ambry Genetics. Exome sequencing is among the commercially available tests.

\section{ACKNOWLEDGEMENTS}

We are grateful to the patients and their families for their participation.
1 Bittles, A. H. Consanguinity and its relevance to clinical genetics. Clin. Genet. 60 89-98 (2001).

2 Stoltenberg, C., Magnus, P., Skrondal, A. \& Lie, R. T. Consanguinity and recurrence risk of birth defects: a population-based study. Am. J. Med. Genet. 82, 423-428 (1999).

3 Majeed-Saidan, M. A., Ammari, A. N., AlHashem, A. M., Al Rakaf, M. S., Shoukri, M. M., Garne, E. et al. Effect of consanguinity on birth defects in Saud women: results from a nested case-control study. Birth Defects Res. A Clin. Mol. Teratol. 103, 100-104 (2014).

4 Teeuw, M., Waisfisz, Q., Zwijnenburg, P. J., Sistermans, E. A., Weiss, M. M., Henneman, L. et al. First steps in exploring prospective exome sequencing of consanguineous couples. Eur. J. Med. Genet. 57, 613-616 (2014).

5 Fahiminiya, S., Almuriekhi, M., Nawaz, Z., Staffa, A., Lepage, P., Ali, R. et al. Whole exome sequencing unravels disease-causing genes in consanguineous families in Qatar. Clin. Genet. 86, 134-141 (2013).

6 Al-Romaih, K. I., Genovese, G., Al-Mojalli, H., Al-Othman, S., Al-Manea, H., Al-Suleiman, M. et al. Genetic diagnosis in consanguineous families with kidney disease by homozygosity mapping coupled with whole-exome sequencing. Am. J. Kidney Dis. 58, 186-195 (2011).

7 Goldenberg-Cohen, N., Banin, E., Zalzstein, Y., Cohen, B., Rotenstreich, Y., Rizel, L. et al. Genetic heterogeneity and consanguinity lead to a 'double hit': homozygous mutations of MYO7A and PDE6B in a patient with retinitis pigmentosa. Mol. Vis. 19, 1565-1571 (2013).

8 Casci, I., Accousti, W. \& Lacassie, Y. Unexpected exome sequencing result: de novo TRPS1 mutation in an infant with infantile scoliosis, mild developmental delay, and history of consanguinity. Am. J. Med. Genet. A 164A, 1334-1337 (2014).

9 Makrythanasis, P., Nelis, M., Santoni, F. A., Guipponi, M., Vannier, A., Bena, F. et al. Diagnostic exome sequencing to elucidate the genetic basis of likely recessive disorders in consanguineous families. Hum. Mutat. 35, 1203-1210 (2014).

10 Ten Kate, L. P., Scheffer, H., Cornel, M. C. \& van Lookeren Campagne, J. G. Consanguinity sans reproche. Hum. Genet. 86, 2 (1991).

11 Alazami, A. M., Patel, N., Shamseldin, H. E., Anazi, S., Al-Dosari, M. S., Alzahrani, F. et al. Accelerating novel candidate gene discovery in neurogenetic disorders via whole-exome sequencing of prescreened multiplex consanguineous families. Cell Rep. 10, 148-161 (2015).

12 Alkuraya, F. S. Impact of new genomic tools on the practice of clinical genetics in consanguineous populations: the Saudi experience. Clin. Genet. 84, 203-208 (2013).

13 Farwell, K. D., Shahmirzadi, L., El-Khechen, D., Powis, Z., Chao, E. C., Davis, B. T. et al. Enhanced utility of family-centered diagnostic exome sequencing with inheritance model-based analysis: results from 500 unselected families with undiagnosed genetic conditions. Genet. Med. 17, 578-586 (2014).

Supplementary Information accompanies the paper on Journal of Human Genetics website (http://www.nature.com/jhg) 